\title{
MedienPädagogik
}

Zeitschrift für Theorie und Praxis der Medienbildung

\section{Gestaltung einer netzbasierten Lernumgebung für einen Fernstudiengang zu „Medien und Informationstechnologien in Erziehung, Unterricht und Bildung“}

\section{Gerhard Tulodziecki}

\section{Einleitung}

In diesem Beitrag stelle ich dar, wie wir in einem kooperativen Verbund eine netzbasierte Lernumgebung für einen Fernstudiengang zu „Medien und Informationstechnologien in Erziehung, Unterricht und Bildung“ gestalten. Das Projekt wird von zwei Arbeitsgruppen an der Universität Paderborn (Allgemeine Didaktik und Medienpädagogik, Didaktik der Informatik) und einer Arbeitsgruppe an der Fernuniversität Hagen (Theorie der Schule und des Unterrichts) getragen. Die Projektleitung teile ich mit Horst Dichanz, Bardo Herzig und Johannes Magenheim.

Der Fernstudiengang basiert auf Entwicklungen zur Lehrerausbildung im Bereich neuer Medien, wie sie im Rahmen der Initiative „Bildungswege in der Informationsgesellschaft (BIG)“ erarbeitet wurden (vgl. u.a. Tulodziecki/Moll/Gallasch 2001). Die - 1995 begonnene - BIG-Initiative geht auf die Bertelsmann Stiftung und Heinz Nixdorf Stiftung zurück. Die jetzige Projektarbeit wird von der ,e-nitiative.nrw.Netzwerk für Bildung“ gefördert.

Das Ministerium für Schule, Wissenschaft und Forschung des Landes Nordrhein-Westfalen hat 1999 eine Rechtsverordnung zum Erwerb der Zusatzqualifikation „Medien und Informationstechnologien in Erziehung, Unterricht und Bildung“ erlassen. Diese basiert auf Empfehlungen einer Arbeitsgruppe, in der Fachvertreterinnen und Fachvertreter der Universitäten Bielefeld, Dortmund und Paderborn mitgewirkt haben. Zielgruppe für die Zusatzqualifikation sind Studierende aller Lehrämter sowie alle, die ein Lehramtsstudium absolviert haben. Der Studienumfang beträgt 30 Semesterwochenstunden (einschließlich eines Praktikums). Die Prüfung für die Zusatzqualifikation kann nach dem ersten Staatsexamen abgelegt werden. Studienleistungen lassen sich allerdings schon während des Lehramtsstudiums erbringen. Ein entsprechender Präsenzstudiengang ist mittlerweile an den Universitäten Paderborn, Dortmund und Münster eingerichtet worden.

Nachdem die Rechtsverordnung vorlag, ergab sich die Frage, ob der Studiengang auch als Fernstudiengang entwickelt werden könnte, um weiteren Interessenten den Zugang zu der Zusatzqualifikation zu ermöglichen. Dazu entwickelten Fachvertreter der Fernuniversität Hagen und der Universität Pa- 
derborn ein Konzept, das im Jahr 2000 verabschiedet wurde. So konnte im Sommersemester 2001 eine zweijährige Pilotphase begonnen werden.

Im Folgenden beschreibe ich zunächst schulische Aufgaben im Medienbereich als Bezugspunkte für den Fernstudiengang. Danach sollen Ziele und Inhalte sowie die Studienbestandteile und der methodische Aufbau des Fernstudiengangs bzw. der netzbasierten Lernumgebung dargestellt werden.

Schulische Aufgaben im Bereich von Medien und Informationstechnologien

Wichtige Bezugspunkte für die Ziele und Inhalte sowie für die methodische Konzeption der netzbasierten Lernumgebung ergeben sich aus den Anforderungen an die Schule im Bereich neuer Medien. Solche Anforderungen sind

- die reflektierte Nutzung von Medien und Informationstechnologien für Lehren und Lernen,

- die Wahrnehmung von medienbezogenen Erziehungs- und Bildungsaufgaben sowie

- die Entwicklung medienpädagogischer Konzepte im Rahmen von Schulprofilen oder Schulprogrammen.

Diese Aufgaben sollen im Folgenden kurz skizziert werden.

\section{Reflektierte Nutzung von Medien und Informationstechnologien}

Wir gehen davon aus, dass das Potential neuer Medien nur dann in sinnvoller Weise genutzt werden kann, wenn es in den Rahmen geeigneter didaktischer Konzepte gestellt wird. Als sinnvoller Rahmen bietet sich ein fall- und handungsorientiertes Vorgehen an. Ein solches Vorgehen kann durch die Konfrontation mit bedeutsamen Erkundungsaufgaben, Problemen, Entscheidungsfällen, Gestaltungs- und Beurteilungsaufgaben angeregt werden (vgl. Tulodziecki 1996a). Eine Erkundung findet z.B. statt, wenn sich Kinder mit Hilfe von Sachbüchern, CD-ROMs und Internet-Recherchen sowie auf der Basis eines Zoo-Besuchs ein Bild von verschiedenen Tieren verschaffen und Informationen zu ihren Lebensräumen zusammenstellen. Die Arbeitsergebnisse lassen sich dann computerbasiert präsentieren. Ein Problem ist z.B. dann gegeben, wenn eine Familie, in der bisher viel Fastfood gegessen wurde, beschließt, ihre Ernährungsgewohnheiten ohne Verlust an Essensgenuss zu verbessern. Um die Aufgabe zu lösen, sind Regeln für eine gesunde Ernährung zu entwickeln. Zeitschriften, Sachbücher und Internet können dazu als Informationsquellen genutzt werden. Ein Rechen- oder Kalkulationsprogramm kann helfen, die Kalorienwerte für ein Frühstück oder andere Mahlzeiten zu berechnen. Im Zusammenhang mit solchen Unterrichtseinheiten lassen sich u.a. unterschiedliche Suchmaschinen für das Internet und ihre informatischen Grundlagen thematisieren. Ein Entscheidungsfall kann z.B. so 
eingeführt werden, dass sich die Lernenden in die Situation einer Regierungskommission in einem Staat versetzen sollen, dessen soziale, politische und ökologische Lage relativ desolat ist. Aufgabe ist es, Entscheidungen zu fällen, z.B. zu notwendigen staatlichen Maßnahmen, die den Zustand des Staats möglichst verbessern sollen. Als Entscheidungshilfe kann unter Umständen ein Simulationsprogramm genutzt werden. Begleitend lässt sich die Frage der Modellbildung und ihrer Umsetzung in Simulationen reflektieren. Eine Gestaltungsaufgabe liegt z.B. vor, wenn sich eine Gruppe von Lernenden entschließt, eine multimediale Homepage zu produzieren. Vorliegende Homepages können dafür zunächst als Gegenstand der Analyse und als Anregung dienen, ehe eine eigene Homepage erstellt wird. Zugleich kann die Frage des möglichen formalen Aufbaus und der Struktur von Internetseiten und ihrer Verknüpfung bearbeitet werden. Eine Beurteilungsaufgabe ist z.B. gegeben, wenn die Lernenden mit Blick auf einen bestimmten Berufsbereich, etwa die Touristikbranche, Aufgaben in Form der Telearbeit simulieren bzw. ausführen. Als „Auftrag“ ließe sich formulieren, für einen Manager, der eine Dienstreise mit einer touristischen Aktivität verbinden möchte, eine interessante und individuelle Reiseroute - auf der Basis von Recherchen im Internet - zusammenzustellen. Eine anschließende Aufgabe könnte darin bestehen, weitere Anwendungsfelder für die Telearbeit zu erkunden und diese in ihrer Bedeutung für Individuum und Gesellschaft zu reflektieren bzw. zu beurteilen.

Aufgaben dieser Art bieten die Chance, die jeweilige Informationsfülle unter spezifischen Aspekten der Aufgabenstellung zu strukturieren und für die Aufgabenlösung nutzbar zu machen. Fähigkeiten zur gezielten Informationssuche, Informationsauswahl und Informationsbewertung können ausgebildet werden. Bei der Bearbeitung lassen sich Medien als Informationsquellen und Lernhilfen, als Werkzeuge und als Objekte der Analyse, als Instrumente für die Präsentation von Arbeitsergebnissen sowie für Kommunikation und Kooperation verwenden.

Wahrnehmung von medienbezogenen Erziehungs- und Bildungsaufgaben

Mit Blick auf Erziehungs- und Bildungsaufgaben im Bereich von Medien und Informationstechnologien gehen wir davon aus, dass Schülerinnen und Schüler in der Schule die Möglichkeit erhalten sollen, Kompetenzen für zwei Handlungszusammenhänge zu erwerben (vgl. Tulodziecki 1997). Der erste Zusammenhang bezieht sich auf eine sinnvolle Nutzung vorhandener Medienangebote, z.B. auf die Verwendung von Fernsehen und Netzangeboten für Information und Lernen, für Unterhaltung und Spiel, für Problemlösung und Entscheidungsfindung, für Kooperation und Kommunikation. Der zweite Zusammenhang ist durch eine eigene Gestaltung medialer Aussagen gekennzeichnet, z.B. die eigene Erstellung einer Zeitung, eines Hörmagazins, eines Videofilms, einer Computersimulation oder einer Homepage. 
Diese Handlungszusammenhänge machen Kenntnisse und Verstehen sowie Analyseund Urteilsfähigkeit in drei inhaltlichen Bereichen notwendig: Zunächst geht es um die Gestaltungsmöglichkeiten, die in Medien Verwendung finden: vom realitätsnahen Foto eines berühmten Bauwerks bis zur grafischen Darstellung der Bevölkerungsentwicklung auf unseren Planeten, von filmischen Gestaltungstechnik bis zu computerbasierten Techniken der Bildbearbeitung. Des Weiteren sind die Nutzungsvoraussetzungen und -Wirkungen von Medien wichtig: von individuellen Einflüssen auf Gefühle, Vorstellungen und Verhaltensorientierungen bis zur Bedeutung der Massen- und Individualkommunikation für die öffentliche Meinungs- und die politische Willensbildung. Schließlich sind die Bedingungen von Medienproduktion und -Verbreitung in den Blick zu nehmen: von technischen Voraussetzungen für die Nutzung von Medien bis zu personalen Bedingungen in einer Rundfunkanstalt, von rechtlichen Bestimmungen zum Datenschutz bis zu wirtschaftlichen Interessen der Computerindustrie und der Netzprovider bzw. der dahinter stehenden Konzerne. Damit ergeben sich insgesamt fünf Aufgabenbereiche für Erziehung und Bildung im Feld von Medien und Informationstechnologien:

- Auswählen und Nutzen von Medienangeboten unter Beachtung von Handlungsalternativen,

- Eigenes Gestalten und Verbreiten von Medienbeiträgen,

- Verstehen und Bewerten von Mediengestaltungen,

- Erkennen und Aufarbeiten von Medieneinflüssen,

- Durchschauen und Beurteilen von Bedingungen der Medienproduktion und Medienverbreitung.

Diese Aufgabenbereiche können helfen, medienpädagogische Aktivitäten in der Schule zu strukturieren (vgl. Tulodziecki/Möller u.a. 1998).

\section{Entwicklung medienpädagogischer Konzepte in der Schule}

Für eine kontinuierliche medienpädagogische Arbeit ist es wichtig, dass verschiedene medienpädagogische Aktivitäten nicht als einmalige und isolierte Aktionen gelten, sondern in einen unterrichtsfachlichen und medienpädagogischen Rahmen gestellt werden (vgl. Tulodziecki u.a. 1995; Dichanz 1998; Spanhel 1999). Um ein geeignetes schulspezifisches Konzept zu entwickeln, empfiehlt es sich, dass sich in der jeweiligen Schule eine Kerngruppe von Lehrerinnen und Lehrern verschiedener Fächer bildet, die sich zunächst mit der Frage auseinander setzt, wie ein inhaltlicher Rahmen für die Medienpädagogik aussehen könnte. Dafür lassen sich die oben genannten fünf Aufgabenbereiche der Medienpädagogik heranziehen.

Auf einer solchen Grundlage sollte die Kerngruppe eine Bestandsaufnahme zu den in der Schule vorhandenen medienpädagogischen Aktivitäten durchführen und diese auswerten. Im nächsten Schritt können medienpäd- 
agogische Unterrichtseinheiten und Projekte für das kommende Schuljahr geplant werden. Dafür sind folgende Grundsätze zu empfehlen:

- Die medienpädagogischen Aktivitäten sollten als kontinuierlicher Prozess über verschiedene Jahrgangsstufen unter Beteiligung verschiedener Fächer oder Lernbereiche konzipiert werden.

- Bei den medienpädagogischen Aktivitäten sollten das gesamte Medienspektrum vom Buch bis zu den neuen Medien - und die altersspezifische Mediennutzung sowie der jeweilige Entwicklungsstand der Kinder berücksichtigt werden.

- Die Auseinandersetzung mit bestimmten Medienangeboten sollte zu exemplarischen Einsichten führen, die auch für andere Medien bedeutsam sind. Zugleich sollen kategoriale Einsichten angestrebt werden, die auch für zukünftige Entwicklungen der Medienlandschaft grundlegend sind.

Wenn anhand der Grundsätze und unter Berücksichtigung der spezifischen Möglichkeiten einer Schule ein Plan für das kommende Schuljahr entwickelt wurde, ist es wichtig, sich - sofern noch nicht vorhanden - um eine geeignete technische und räumliche Infrastruktur zu bemühen. Des Weiteren sind Qualifizierungsmaßnahmen notwendig. Der Entwicklungsprozess sollte schließlich in ein medienpädagogisches Konzept - als Teil des Schulprofils bzw. Schulprogramms - einmünden.

\section{Ziele und Inhalte der netzbasierten Lernumgebung}

Die Ziele und Inhalte für die netzbasierte Lernumgebung zu „Medien und Informationstechnologien in Erziehung, Unterricht und Bildung“ ergeben sich zum einen aus den allgemeinen Zielen für die universitäre Lehrerbildung und zum anderen aus den besonderen Erfordernissen im Bereich von Medien und Informationstechnologien.

\section{Allgemeiner Zielrahmen}

Die universitäre Lehrerbildung zielt schwerpunktmäßig auf eine wissenschaftliche Fundierung für berufliche Aufgaben. Zum einen sollen Studierende im Lehramtsbereich die wissenschaftlichen Grundlagen für die Wahrnehmung von Unterrichts- bzw. Bildungsaufgaben sowie von Erziehungs- und Schulentwicklungsaufgaben erwerben. Zum anderen geht es darum, eine forschende Grundhaltung aufzubauen und Forschungsergebnisse sowie Forschungsmethoden auf berufliche Aufgaben zu beziehen. Begleitend soll die Persönlichkeitsentwicklung der Studierenden gefördert werden.

Damit ist ein allgemeiner Kontext für die Auseinandersetzung mit medienpädagogischen Fragen markiert. 
Ziele für die Lehrerbildung im Bereich von Medien und Informationstechnologien Medienverwendung und Mediengestaltung, Medienerziehung und Medienbildung als Aufgaben der Schule setzen medienpädagogische Kompetenzen auf Seiten der Lehrerinnen und Lehrer voraus. Demgemäss geht es in der Lehrerbildung darum, Möglichkeiten für den Erwerb medienpädagogischer Kompetenzen zu schaffen.

Die medienpädagogische Qualifizierung sollte zunächst darauf gerichtet sein, die jeweils eigene Medienkompetenz zu stärken. Medienkompetenz umfasst die angesprochenen fünf Aufgabenbereiche der Medienpädagogik und schließt technische Fertigkeiten der Handhabung der Medien ein. Über die Stärkung der eigenen (allgemeinen) Medienkompetenz hinaus geht es um die Fähigkeit, die Bedeutung von Medien für Kinder und Jugendliche in sensibler Weise zu erfassen und als Ausgangssituation des Lernens mit oder über Medien zu berücksichtigen. Des Weiteren sollen Lehrerinnen und Lehrer in der Lage sein, Medienangebote in ihrem Unterricht in reflektierter Weise zu verwenden, d.h. Medienangebote für ihre Fächer nach lernrelevanten Kriterien zu analysieren und auszuwählen sowie Konzepte für die Verwendung von Medien im Rahmen geeigneter Lehr- und Lernformen zu erarbeiten und umzusetzen. Außerdem ist die Fähigkeit wichtig, Medienthemen in angemessener Weise zu behandeln, d.h. die Bedeutung von Medien für inhaltliche und methodische Fragen des jeweiligen Faches zu bedenken sowie medienbezogene Erziehungs- und Bildungsprozesse bei den Schülerinnen und Schülern zu initiieren und zu begleiten. Schließlich sollte angestrebt werden, personale und institutionelle Bedingungen für medienpädagogische Umsetzungen in der Schule zu durchschauen, d.h. die Bedeutung der Medien für Fragen der Professionalität des Lehrberufs zu reflektieren, schulische Bedingungen von Medienverwendung und Mediengestaltung sowie Medienerziehung und Medienbildung zu bedenken sowie Ideen für die schulische Umsetzung zu entwickeln und zu realisieren.

Damit ergeben sich für die Lehrerbildung fünf Zielbereiche (vgl. Tulodziecki 1996b; MSWWF 2000; Blömeke 2000):

- Stärkung der Medienkompetenz, einschließlich einer angemessenen technischen Handhabung von Medien und Informationstechnologien,

- Erwerb von Kenntnissen zur und Sensibilität für die Bedeutung von Medien für Kinder und Jugendliche,

- Befähigung zur reflektierten Nutzung von Medien und Informationstechnologien für Lehren und Lernen,

- Befähigung zur Wahrnehmung von Erziehungs- und Bildungsaufgaben im Bereich von Medien und Informationstechnologien,

- Befähigung zur Mitwirkung an der Gestaltung medienpädagogischer Konzepte in der Schule. 
Dieser Zielkatalog beschreibt einen allgemeinen Rahmen für die Lehrerbildung im Bereich von Medien und Informationstechnologien. In diesem Rahmen bietet der Fernstudiengang die Möglichkeit, die eigene Medienkompetenz weiterzuentwickeln, wissenschaftliche Grundlagen für die verschiedenen Felder medienpädagogischer Kompetenz zu erwerben sowie Umsetzungen zu entwerfen, zu erproben und zu reflektieren. Der Zielrahmen gilt dabei sowohl für die Erziehungswissenschaft als auch für die Fachdidaktiken und für die Fachwissenschaften.

\section{Inhalte}

Das Lehrangebot für die Zusatzqualifikation bzw. den Fernstudiengang umfasst im Kern die folgenden fünf Module (A bis D) mit den angegebenen Teilgebieten (der dabei verwendete Medienbegriff umfasst sowohl Medienprodukte als auch Werkzeuge und Kommunikationsdienste):

A Medienwissenschaftliche und informatische Grundlagen

A 1 Analyse und Kritik von Medien

A 2 Auswahl und Nutzung von Medien

A 3 Gestaltung und Verbreitung von Medien

A 4 Informatische Grundlagen der Medienverwendung und Mediengestaltung

B Mediennutzung für Lehren und Lernen

B 1 Erziehungswissenschaftliche Grundlagen der Medienverwendung in Lehr- und Lernprozessen

B 2 Fachbezogene und fächerübergreifende Medienverwendung

C Erziehungs- und Bildungsaufgaben im Bereich von Medien und Informationstechnologien

C 1 Grundlagen zu medienbezogenen Erziehungs- und Bildungsaufgaben

C 2 Fachbezogene und fächerübergreifende Wahrnehmung medienbezogener Erziehungsund Bildungsaufgaben

D Entwicklung medienpädagogischer Konzepte in Bildungseinrichtungen

D 1 Medienpädagogik und Profilentwicklung

D 2 Medienorganisation und Medienvemetzung

Die Teilgebiete werden im Fernstudiengang - bis auf die Teilgebiete B 2 und C 2 - als Pflichtveranstaltungen angeboten. Für die Teilgebiete B 2 und C 2 stehen im Fernstudiengang verschiedene fachdidaktische Angebote als Wahlpflichtveranstaltungen zur Verfügung. Außerdem umfasst das Studienangebot eine Einführung als Pflichtveranstaltung und einen Wahlbereich. Das Angebot pro Teilgebiet entspricht jeweils einem Studienumfang von 2 bis 4 Semesterwochenstunden. Jedes Teilgebiet umfasst mehrere Kurseinheiten. 


\section{Studienbestandteile und methodische Konzeption}

Die methodische Konzeption des Fernstudienangebots bzw. der netzbasierten Lernumgebung ist auf einen fall- und handlungsorientierten selbstgesteuerten Lernprozess ausgerichtet, der je nach Bedarf der Studierenden mit kommunikativen Möglichkeiten verbunden werden kann.

\section{Studienbestandteile}

Das fall- und handlungsorientierte Vorgehen soll vor allem durch die verschiedenen Kurseinheiten bzw. Studienmaterialien angeregt und unterstützt werden. Als Hilfen bieten wir Hinweise zum Vorgehen, einen Index und ein Glossar. Die Studienmaterialien liegen netzbasiert und/oder in schriftlicher Form vor. Die kommunikativen Möglichkeiten umfassen Ankündigungen, Foren und Chats in der netzbasierten Lernumgebung sowie einen in der Planung befindlichen Kooperations- und Präsentationsbereich. Darüber hinaus gibt es Präsenzangebote. Schließlich gehören Lernerfolgskontrollen in Form von Einsendeaufgaben, Klausuren, Seminararbeiten mit medialem Produkt sowie eine Abschlussarbeit mit Disputation zum Fernstudiengang (vgl. auch http://www.fernunihagen.de/festum/). Eine Übersicht über die verschiedenen Studienbestandteile zeigt die Darstellung 1. Elemente der netzbasierten Lernumgebung sind in diesem Rahmen kursiv gekennzeichnet.

\begin{tabular}{llll}
\hline $\begin{array}{l}\text { Studienbestandteile } \\
\text { Studienmaterialien }\end{array}$ & Lernhilfen & Kommunikation & Lernerfolgskontrollen \\
\hline $\begin{array}{l}\text { Kurseinheiten/ Kurs- } \\
\text { abschnitte netzbasiert }\end{array}$ & $\begin{array}{l}\text { Hinweise zum Vorge- } \\
\text { hen Ankündigungen }\end{array}$ & Ankündigungen & $\begin{array}{l}\text { Ausarbeitungen zu } \\
\text { Einsendeaufgaben }\end{array}$ \\
$\begin{array}{l}\text { Kurseinheiten/ Kurs- } \\
\text { abschnitte gedruckt }\end{array}$ & Index & Foren & Klausuren \\
& Glossar & Chats & Seminararbeiten mit \\
& & $\begin{array}{l}\text { Kooperations- und } \\
\text { Präsentationsbereich }\end{array}$ & Produkt \\
& & Praktikumsbericht \\
& & Abschlussarbeit mit \\
Praktikum und Prä- & & Disputation \\
senzphasen & & \\
\hline
\end{tabular}

Darst. 1: Übersicht über die Studienbestandteile für den Fernstudiengang „Medien und Informationstechnologien in Erziehung, Unterricht und Bildung“. 
Methodischer Aufbau der Kurseinheiten und Kursabschnitte

Die Kurseinheiten sind auf die oben genannten Teilgebiete bezogen. Beispielsweise umfasst das Teilgebiet „Erziehungswissenschaftliche Grundlagen der Medien Verwendung in Lehr- und Lernprozessen“ die folgenden Kurseinheiten:

- Lerntheoretische und mediendidaktische Grundlagen der Medienverwendung und Mediengestaltung

- Empirische Forschung zum Einsatz von Medien im Unterricht

- Medienverwendung und Mediengestaltung für handlungsorientierte Lehr- und Lernprozesse

Die zuletzt genannte Kurseinheit enthält beispielsweise die folgenden Kursabschnitte:

- Merkmale handlungsorientierter Lehr-Lernprozesse,

- Funktionen von Medien in handlungsorientierten Lehr-Lernprozessen,

- Vorbereitung eines handlungsorientierten Unterrichts mit Medienverwendung,

- Analyse und Bewertung von Unterrichtseinheiten und Projekten mit Medienverwendung.

Die Kursabschnitte sind so aufgebaut, dass eine fall- und handlungsorientierte Bearbeitung möglich wird. Um eine solche anzuregen und zu unterstützen, enthält jeder Kursabschnitt nach einleitenden Hinweisen am Beginn eine Aufgabe, in der ein praxisbezogenes Problem, ein relevanter Entscheidungsfall, eine bedeutsame Gestaltungsaufgabe oder eine wichtige Analyse- bzw. Beurteilungsaufgabe eingeführt wird. Wir bevorzugen dabei Aufgaben bzw. Fälle, die einen mittleren Komplexitätsgrad aufweisen und zusammen mit der Erarbeitung der notwendigen Informationen sowie einer Formulierung der Aufgabenlösung einen Zeitumfang von 2 bis 4 Stunden verlangen. Beispielsweise wird den Studierenden in dem Kursabschnitt „Vorbereitung eines handlungsorientierten Unterrichts mit Medienverwendung“ ein konkretes Medienangebot - hier die CD-ROM „Die Alpen“ - vorgestellt. Danach werden sie mit der Aufgabe konfrontiert, für die Arbeit mit dieser CD-ROM einen problem-, entscheidungs-, gestaltungs- oder beurteilungsorientierten Unterrichtsablauf zu entwerfen. Ein weiteres Beispiel: In dem Kursabschnitt „Analyse und Bewertung von Unterrichtseinheiten und Projekten mit Medienverwendung“ wird zunächst ein Projekt skizziert, bei dem Schülerinnen und Schüler eine eigene Werbeanzeige für ein von ihnen bestimmtes Produkt gestalten. Die Aufgabe für die Studierenden besteht nun darin, dieses Projekt hinsichtlich unterschiedlicher Kriterien zu analysieren und zu bewerten und ggf. Verbesserungsvorschläge zu machen.

Nachdem die Studierenden zu der jeweiligen einführenden Aufgabe erste Lösungsansätze bedacht und damit ihre Vorkenntnisse aktiviert haben, werden grundlegende Informationen angeboten. Bezogen auf das erste Beispiel 
enthalten die grundlegenden Informationen eine Systematik vorbereitender Überlegungen für die Planung von Unterricht mit computerbasierten Medienangeboten. Beim zweiten Beispiel wird in den grundlegenden Informationen ein Kriterienkatalog für die Analyse und Bewertung von Unterrichtseinheiten und Projekten mit Medien Verwendung entwickelt.

Auf der Basis der grundlegenden Informationen sollen sich die Studierenden dann wieder der einführenden Aufgabe zuwenden, ihre spontanen Lösungsansätze überdenken und die einführende Aufgabe in differenzierter Weise bearbeiten. So können sie im ersten Beispiel einen fundierten Unterrichtsentwurf mit der Verwendung eines computerbasierten Medienangebots entwickeln und im zweiten Beispiel das Projekt kriterienbezogen analysieren und bewerten sowie Verbesserungsvorschläge erarbeiten. Ihre Lösungen sollen die Studierenden dann jeweils mit einem vorgegebenen Lösungskommentar vergleichen.

Zur Erweiterung und Ergänzung sowie für eigene vertiefende Erkundungen werden den Studierenden dann für jeden Abschnitt Literaturempfehlungen und Hinweise auf interessante Internetadressen gegeben.

Die einzelnen Kursabschnitte bilden den Kern der netzbasierten Lernumgebung und sind somit jeweils folgendermaßen gegliedert:

- Einleitende Hinweise und Teilziele

- Einführende Aufgabe

- Grundlegende Informationen

- Lösungskommentar

- Literaturempfehlungen

- Hinweise auf Internetadressen

- Liste der zitierten Literatur

Im weiteren Verlauf der Entwicklung sollen möglichst alle Teilgebiete der netzbasierten Lernumgebung mit ihren Kurseinheiten und Kursabschnitten in dieser Form über das Netz verfügbar und miteinander verbunden bzw. „verlinkt“ sein. Der Zugang zu einem Thema wird zwar jeweils über die einleitenden Hinweise und einführenden Aufgaben empfohlen, kann jedoch auch über die grundlegenden Informationen erfolgen. Auch ein direkter Zugang zu den Literaturempfehlungen, zur zitierten Literatur sowie zu den Internetadressen für die einzelnen Themen ist möglich.

\section{Schlussbemerkung}

Wir sehen in der fall- bzw. handlungsorientierten Strukturierung der einzelnen Kursabschnitte einen wichtigen Schritt zu einer angemessenen Verbindung lerntheoretischen und didaktischen Forderungen an Lehr-Lernprozesse sowie zu einer sinnvollen Verknüpfung von Instruktion und Konstruktion. So lassen sich Lernprozesse anregen und unterstützen, die letztlich zu einem theoriebasierten und anwendungsfähigen sowie praxisrelevanten Wissen führen sollen. 


\section{Literatur}

Blömeke, S.: Medienpädagogische Kompetenz. Theoretische und empirische Fundierung eines zentralen Elements der Lehrerausbildung. München 2000.

Dichanz, H. (Hrsg.): Schulprofil „Medienerziehung“. Handreichungen zur Entwicklung eines medienspezifischen Schulprofils. Halle/Saale: Landesrundfunkausschuss für Sachsen-Anhalt 1998.

Ministerium für Schule und Weiterbildung, Wissenschaft und Forschung des Landes NordrheinWestfalen (Hrsg.): Zukunft des Lehrens - Lernen für die Zukunft: Neue Medien in der Lehrerausbildung. Rahmenkonzept. Frechen 2000.

Spanhel, D.: Integrative Medienerziehung in der Hauptschule. Ein Entwicklungsprojekt auf der Grundlage responsiver Evaluation. München1999.

Tulodziecki, G.: Unterricht mit Jugendlichen. Eine handlungsorientierte Didaktik mit Unterrichtsbeispielen. Bad Heilbrunn 1996a.

Tulodziecki, G.: Lehrerfortbildung im Bereich neuer elektronischer Medien. In: Bertelsmann Stiftung (Hrsg.): Neue Medien in den Schulen. Projekte - Konzepte - Kompetenzen. Eine Bestandsaufnahme. Gütersloh 1996b, S. 165-182.

Tulodziecki, G.: Medien in Erziehung und Bildung. Grundlagen und Beispiele einer handlungs- und entwicklungsorientierten Medienpädagogik. Bad Heilbrunn 1997.

Tulodziecki, G./Gallasch, U./Moll, St.: Fallbeispiele für die Verankerung neuer Medien in der Lehrerausbildung. In: Bentlage, U./Hamm, I. (Hrsg.): Lehrerausbildung und neue Medien. Erfahrungen und Ergebnisse eines Hochschulnetzwerkes. Gütersloh 2001, S. 107-122.

Tulodziecki, G./Möller, D. u.a.: Rahmen für die Medienerziehung in der Sekundarstufe I. Ergebnisse des Modellversuchs „Differenzierte Medienerziehung als Elemente allgemeiner Bildung“. Düsseldorf: Ministerium für Schule und Weiterbildung, Wissenschaft und Forschung des Landes Nordrhein-Westfalen 1998.

Tulodziecki, G. u.a.: Handlungsorientierte Medienpädagogik in Beispielen. Projekte und Unterrichtseinheiten für Grundschulen und weiterführende Schulen. Bad Heilbrunn 1995. 